\title{
Association of HPV infection and clearance with cervicovaginal immunology and the vaginal microbiota
}

\author{
B Shannon ${ }^{1,2}$, TJ Yi ${ }^{1,2}$, S Perusini ${ }^{3}, \mathrm{P} \mathrm{Gajer}^{4,5}, \mathrm{~B} \mathrm{Ma}^{4,5}$, MS Humphrys ${ }^{4,5}$, J Thomas-Pavanel ${ }^{6}$, L Chieza ${ }^{6}$, \\ P Janakiram $^{6}$, M Saunders ${ }^{6}$, W Tharao $^{6}$, S Huibner ${ }^{1}$, K Shahabi ${ }^{1}$, J Ravel ${ }^{4,5}$, A Rebbapragada ${ }^{3,7}$ and \\ R Kaul ${ }^{1,2,8}$
}

Cervical human papillomavirus (HPV) infection may increase HIV risk. Since other genital infections enhance HIV susceptibility by inducing inflammation, we assessed the impact of HPV infection and clearance on genital immunology and the cervico-vaginal microbiome. Genital samples were collected from 65 women for HPV testing, immune studies and microbiota assessment; repeat HPV testing was performed after 6 months. All participants were HIV-uninfected and free of bacterial STIs. Cytobrush-derived T cell and dendritic cell subsets were assessed by multiparameter flow cytometry. Undiluted cervico-vaginal secretions were used to determine cytokine levels by multiplex ELISA, and to assess bacterial community composition and structure by 16S rRNA gene sequence analysis. Neither HPV infection nor clearance were associated with broad differences in cervical T cell subsets or cytokines, although HPV clearance was associated with increased Langerhans cells and HPV infection with elevated IP-10 and MIG. Individuals with HPV more frequently had a high diversity cervico-vaginal microbiome (community state type IV) and were less likely to have an L. gasseri predominant microbiome. In summary, HPV infection and/or subsequent clearance was not associated with inflammation or altered cervical Tcell subsets, but associations with increased Langerhans cells and the composition of the vaginal microbiome warrant further exploration.

\section{INTRODUCTION}

Human papillomavirus (HPV) is the most common sexually transmitted infection (STI) globally. ${ }^{1}$ The majority of HPV infections are cleared quickly by the host, ${ }^{2}$ but high-risk subtypes (HR-HPV) can persist and cause cervical and other types of cancer. ${ }^{3}$ Recent meta-analysis has associated HPV infection with a two-fold increased risk of HIV acquisition, ${ }^{4}$ possibly because of the recruitment of cells highly-susceptible to HIV infection (HIV target cells) or other changes in the immunology and composition of bacterial communities within the genital tract. ${ }^{5}$

Many STIs induce robust host mucosal immune responses and genital inflammation, ${ }^{6}$ in order to facilitate pathogen clearance and in some cases protect against re-infection. ${ }^{7}$ However, this inflammatory response may render individuals more susceptible to HIV infection. Not only do activated CD4 T cells express elevated levels of the HIV co-receptor CCR $5,{ }^{8}$ making them preferential HIV targets, ${ }^{9}$ but STIs also increase pro-inflammatory cytokine levels and neutrophil protease levels ${ }^{10-12}$ which are associated with perturbed epithelial cell differentiation, cell-cell contacts, and epithelial barrier function and integrity. ${ }^{11}$ In keeping with this, macaque models have shown that levels of mucosal CCR5 ${ }^{+}$[ref. 13] CD4 T cells are predictive of subsequent SIV infection risk, and HIV acquisition has been linked to increased genital pro-inflammatory cytokines in human cohorts. ${ }^{14,15}$

${ }^{1}$ Departments of Medicine, University of Toronto, Toronto, Ontario, Canada. ${ }^{2}$ Departments of Immunology, University of Toronto, Toronto, Ontario, Canada. ${ }^{3}$ Public Health Ontario_-Toronto Public Health Laboratory, Toronto, Ontario, Canada. ${ }^{4}$ Institute for Genome Sciences, University of Maryland School of Medicine, Baltimore, Maryland, USA. ${ }^{5}$ Department of Microbiology and Immunology, University of Maryland School of Medicine, Baltimore, Maryland, USA. ${ }^{6}$ Women's Health in Women's Hands Community Health Centre, Toronto, Ontario, Canada. ${ }^{7}$ Laboratory Medicine and Pathobiology, University of Toronto, Toronto, Ontario, Canada and ${ }^{8}$ University Health Network, Toronto, Ontario, Canada. Correspondence: B Shannon (brett.shannon@utoronto.ca) or R Kaul (rupert.kaul@utoronto.ca) 
Classical bacterial STIs such as gonorrhea and chlamydia cause genital inflammation ${ }^{14}$ and an increase in activated mucosal CD $4{ }^{+}$T cells, ${ }^{16}$ and have been associated with a 3-5 fold increase in HIV acquisition. Interestingly, although herpes simplex virus type-2 (HSV-2) is not associated with elevated pro-inflammatory genital cytokines, ${ }^{17}$ HSV-2 infection has been consistently associated with elevated levels of activated $\mathrm{CD}^{+}{ }^{+} \mathrm{T}$ cells in the genital mucosa, ${ }^{18,19}$ and also with a threefold increase in HIV acquisition risk. ${ }^{17}$ Even in the absence of classical STIs, HIV risk is increased $60 \%$ in the context of bacterial vaginosis (BV), defined as the absence of vaginal Lactobacillus spp. and an increase in a wide array of strict and facultative anaerobic bacteria. ${ }^{20}$ Further, BV-associated bacteria have been associated with genital inflammation. ${ }^{10,14}$ However, the mucosal immune impact of HPV has not been well defined, despite its high global prevalence.

Limited data have linked host immune clearance of HPV with a Th1 pro-inflammatory response in the female genital tract, ${ }^{5}$ with increased dendritic cell density in the foreskin, ${ }^{21}$ and with peripheral blood CD8 $\mathrm{T}$ cell responses to HPV proteins in vitro. ${ }^{22}$ These observations led us to hypothesize that an inflammatory host mucosal immune response to HPV is necessary for host immune clearance, but that this inflammatory response would recruit highly HIV-susceptible T cells to the genital tract, thereby enhancing HIV susceptibility.
In Ontario, the African/Caribbean and other black (ACB) community bears a disproportionate burden of HIV infection, ${ }^{23}$ and also has a high prevalence of HPV infection. ${ }^{23}$ Therefore, we performed a longitudinal observational study in ACB women to define the mucosal immune impact of prevalent $\mathrm{HPV}$ infection and subsequent host immune clearance. The primary study endpoints were the overall number of endocervical $\mathrm{CD}^{+}{ }^{+} \mathrm{T}$ cells, as well as of $\mathrm{CD} 4^{+} \mathrm{T}$ cell subsets expressing CCR5 and the immune activation markers CD69 and CD38/HLA-DR. Analyses compared HPV uninfected and infected women, as well as women who went on to clear HPV in the short term vs. maintaining persistent HPV infection. Differences in the absolute number of various dendritic cell (DC) subsets, cervico-vaginal levels of pro-inflammatory and chemoattractant cytokines, and the associated composition and structure of the vaginal microbiota were also assessed.

\section{RESULTS}

\section{Participant demographics}

A total of 65 African/Caribbean women were recruited and enrolled into the study. Insufficient cells were recovered from cytobrushes for six participants, and these participants were excluded from subsequent analysis. Of the remaining 59 participants, 36 (61.0\%) were HPV negative and $23(39.0 \%)$ were HPV positive at the time of baseline sampling, with $13 / 23$

Table 1 Participant demographics, by HPV status (total $n=59$ )

\begin{tabular}{|c|c|c|c|c|c|c|}
\hline Variables & $\begin{array}{l}\text { HPV Negative } \\
\qquad(n=36)\end{array}$ & $\begin{array}{l}\text { HPV Positive } \\
\quad(n=23)\end{array}$ & $\begin{array}{c}\boldsymbol{P} \\
\text { value }^{\mathrm{a}}\end{array}$ & $\begin{array}{l}\text { HR-HPV Posi- } \\
\text { tive }(n=13)^{b}\end{array}$ & $\begin{array}{l}\text { Clear HPV } \\
(n=11)^{\mathrm{b}}\end{array}$ & $\begin{array}{l}\text { Persist HPV } \\
(n=8)^{\mathrm{b}} \\
\end{array}$ \\
\hline \multicolumn{7}{|l|}{ Demographics } \\
\hline Age (y; (IQR)) & $37.5(28.5-46.5)$ & $33(27.0-39.0)$ & 0.250 & 37 (25.5-48.5) & $37(33-41)$ & $29(20.5-37.5)$ \\
\hline Postmenopausal (n (\%)) & $6(16.7)$ & $2(8.7)$ & 0.464 & $2(15.4)$ & $1(9.1)$ & $1(12.5)$ \\
\hline $\begin{array}{l}\text { Time since menses } \\
(\mathrm{d} ;(\mathrm{QQR}))^{\mathrm{c}}\end{array}$ & $15(12-17)$ & $15(12.5-16)$ & 0.530 & $15(13-16)$ & $15.5(12.75-16.25)$ & $14(10-15)$ \\
\hline \multicolumn{7}{|l|}{ Behavioral characteristics } \\
\hline Sex in the past week (n (\%)) & $13(36.1)$ & $10(43.5)$ & 0.596 & $4(30.8)$ & $5(45.5)$ & $4(50.0)$ \\
\hline $\begin{array}{l}\text { Days since intercourse } \\
(\mathrm{y} ;(\mathrm{IQR}))\end{array}$ & $3(1-6)$ & $5(3-7)$ & 0.238 & $4.5(2-6.5)$ & $6(3-7)$ & $6(3-7)$ \\
\hline Condom use (n (\%)) & $2(15.4)$ & $7(70.0)^{d}$ & 0.013 & $1(25.0)$ & $2(40.0)$ & $2(25.0)$ \\
\hline $\begin{array}{l}\text { Hormonal contraceptive } \\
\text { use }(n(\%))\end{array}$ & $5(13.9)$ & $1(4.3)$ & 0.389 & $0(0)$ & $1(9.1)$ & $0(0)$ \\
\hline Intravaginal practices (n (\%)) & $4(11.1)$ & $3(13.0)$ & 1.000 & $2(15.4)$ & $0(0)$ & $3(37.5)$ \\
\hline \multicolumn{7}{|l|}{ Clinical characteristics (n (\%)) } \\
\hline $\begin{array}{l}\text { Bacterial vaginosis } \\
\text { (Nugent } \geqslant 7 \text { ) }\end{array}$ & $2(5.6)$ & $5(21.7)$ & 0.098 & $3(23.1)$ & $2(18.2)$ & $2(25.0)$ \\
\hline HSV-1 & $36(100)$ & $18(78.3)^{d}$ & 0.007 & $11(84.6)^{d}$ & $9(81.8)^{d}$ & $5(62.5)$ \\
\hline HSV-2 & $22(61.1)$ & $16(69.6)$ & 0.585 & $11(84.6)$ & $8(72.7)$ & $5(62.5)$ \\
\hline Yeast & $4(11.1)$ & $2(8.7)$ & 0.765 & $0(0)$ & $1(9.1)$ & 1 (12.5) \\
\hline
\end{tabular}

${ }^{a} P$ value associated with the HPV Negative vs. HPV Positive comparison.

'These groups represent sub-groups of the "HPV Positive" group.

${ }^{\mathrm{c}}$ Among women reporting regular menstrual cycles.

${ }^{d} P<0.05$ vs. HPV Negative group (except those with persistent HPV which were compared with those who cleared HPV). 
(56.5\%) infected with at least one HR-HPV type. Among HPVinfected participants, $21 / 23(91.3 \%)$ attended the 6 month follow up visit; 11/21 (52.4\%) were HPV negative (clearance group), $8 / 21$ (38.1\%) were infected with the same HPV type(s) (persistent group), and 2/21 (9.5\%) had cleared one HPV subtype but were newly or persistently infected by another subtype (excluded from analyses of clearance vs. persistence). Among HPV negative participants, 30/36 (83.3\%) attended the 6 month follow up visit 24/30 (80.0\%) remained HPV negative and $6 / 30(20.0 \%)$ acquired an HPV infection.

There were no differences between the HPV negative and HPV positive groups in terms of age (median 37.5 years, IQR 28.5-46.5; vs. 33 years; IQR 27.0-39.0) or number of women reporting regular menstrual cycles $(30 / 36 ; 83.3 \%$ vs. $21 / 23$; 91.3\%; Table 1), nor were there differences in the number reporting sexual intercourse in the past week $(13 / 36 ; 36.1 \%$ vs. $10 / 23 ; 43.5 \%)$. Among those sexually active in the past week the median time since previous intercourse was similar ( $3 ; 1-6$ vs. 5 ; 3-7 days), although HPV-uninfected women were less likely to have used a condom $(2 / 13 ; 15.4 \%$ vs. $7 / 10 ; 70.0 \%, P=0.008)$. There were similar rates of hormonal contraceptive use and intravaginal washing between the HPV negative and positive participants $(5 / 36 ; 13.9 \%$ vs. $1 / 23 ; 4.3 \%$ and $4 / 36 ; 11.1 \%$ vs. $3 / 23 ; 13.0 \%$, respectively). In terms of genital co-infections, the prevalence of BV and HSV-2 was similar amongst HPV negative and positive women $(2 / 36,5.6 \%$ vs. $5 / 23,21.7 \%$, $P=0.098$; and $22 / 36,61.1 \%$ vs. $16 / 23,69.6 \%, P=0.585$, respectively); however HPV negative women had higher rates of HSV-1 infection than their HPV positive counterparts (36/36; $100 \%$ vs. $18 / 23 ; 78.3 \%, P=0.003)$. Yeast detected on vaginal smears also did not differ between HPV positive (2/23; $8.7 \%)$ and HPV negative participants $(4 / 36 ; 11.1 \%, P=0.765)$. No participants were infected with HIV-1/2, N. gonorrhoeae, or C. trachomatis.

Among the HPV positive participants, those infected with HR-HPV and those who went on to clear HPV infection had a lower prevalence of HSV-1 infection than HPV negative participants $(11 / 13 ; 84.6 \%$ vs. $36 / 36 ; 100 \%, P=0.016$ and $9 / 11$; $81.8 \%$ vs. $36 / 36 ; 100 \%, P=0.009$, Table 1$)$. No other demographic factors that were assessed varied between HPV negative and HPV positive/HR-HPV positive/those that cleared HPV infection, nor were there any differences between those with persistent HPV infection and those who cleared HPV.

\section{HPV infection and immune cell subsets}

The overall endocervical CD4 T cell number did not differ between HPV negative participants (mean $1153 \mathrm{CD} 4{ }^{+} \mathrm{T}$ cells/ cytobrush) and HPV positive participants ( 889 cells/cytobrush, $P=0.804$ ), or the subset of participants infected by HR-HPV (863 cells/cytobrush, $P=0.930$ ). Furthermore, among participants with baseline HPV infection, cervical $\mathrm{CD} 4^{+} \mathrm{T}$ cells numbers were similar in those who cleared HPV over the next 6 months (970 cells per cytobrush, $P=0.459$ ) and those with persistent HPV infection (988 cells per cytobrush; $P=0.265$, Figure 1a). There were no associations observed in the female genital tract or blood between HPV infection and the proportion or number of $\mathrm{CD}^{+} \mathrm{T}$ cells expressing the activation markers CD69 (early activation) or CD38/HLADR (chronic activation), or the mucosal homing integrin $\alpha 4 \beta 7$ (Figure 1b-e).

Interestingly, participants who cleared HPV had a higher absolute number of endocervical Langerhans cells (5018 cells per cytobrush) than both HPV negative women (635 cells per cytobrush, $P=0.015$ ) and those with persistent HPV infection (180 cells per cytobrush, $P=0.023$, Figure 2a). However, no association with prevalent HPV infection was observed: HPV positive (2488 cells per cytobrush) and negative (635 cells per cytobrush) participants had similar numbers of endocervical Langerhans cells $(P=0.608)$. Further, there were no differences in the absolute numbers of mDCs or monocytes (Figure $\mathbf{2 b}$ and $\mathbf{c}$ ), nor were there differences in the expression of C-type lectin receptors (mannose receptor/DC-SIGN) on these subsets between groups (data not shown).

\section{HPV infection and soluble mediators}

Having observed no association between HPV infection status and $\mathrm{T}$ cell subsets in the female genital tract, we explored potential associations between HPV infection or clearance and pro-inflammatory cytokine levels in cervicovaginal secretions (median mass collected by Instead Softcup $0.26 \mathrm{~g}$, range 0.09 $1.36 \mathrm{~g}$ ). To reduce concerns around multiple comparisons, our primary endpoint was the presence or absence of elevated proinflammatory cytokines, using a pre-specified aggregate score in which genital inflammation was defined as having at least $3 / 7$ of the following cytokines in the upper quartile for all participants: IL- $1 \alpha$, IL- $1 \beta$, IL- 8 , MIP- $1 \beta$, MIP- $3 \alpha$, RANTES, and TNF- $\alpha^{11}$

Using this aggregate score, $16 / 59$ participants (27.1\%) were defined as having genital inflammation. No association with genital inflammation was observed between those with prevalent HPV infection $(8 / 23 ; 34.8 \%)$ vs. HPV negative participants $(8 / 36 ; 22.2 \%, P=0.371)$, neither was genital inflammation associated with those who subsequently cleared HPV infection $(4 / 11 ; 36.4 \%)$, those with persistent HPV infection $(3 / 8 ; 37.5 \%, P=0.960)$, or HPV negative participants $(8 / 36 ; 22.2 \%, P=0.435)$. In addition, no associations were observed between prevalent or clearance of HPV infection and individual pro-inflammatory cytokines measured (IL- $1 \alpha$, IL-1 $\beta$, IL-6, IL-10, IL-17, RANTES, and TNF $\alpha$ Figure 3a). Although levels of the chemokines MIG and IP-10 (which were not included in the aggregate inflammation score) were significantly higher in participants with prevalent HPV infection (MIG mean $3.5 \log 10$ and IP-10 mean $2.6 \log \mathrm{pg} \mathrm{mL}^{-1}$ ) as compared with HPV negative participants (MIG mean 3.2log10 and IP-10 mean $2.4 \log \mathrm{pg} \mathrm{mL}-1, P=0.009$ and $P=0.034$, respectively, Figure $\mathbf{3 b}$ ), other chemokine levels (GM-CSF, IL-8, MCP-1, MIP-3 $\alpha$, and MIP-1 $\beta$ ) were not associated with prevalent HPV infection or subsequent clearance.

\section{HPV infection and the vaginal microbiota}

Samples for 8 participants were unavailable for 16S rRNA gene sequencing. The cervico-vaginal microbiota of the remaining 
a

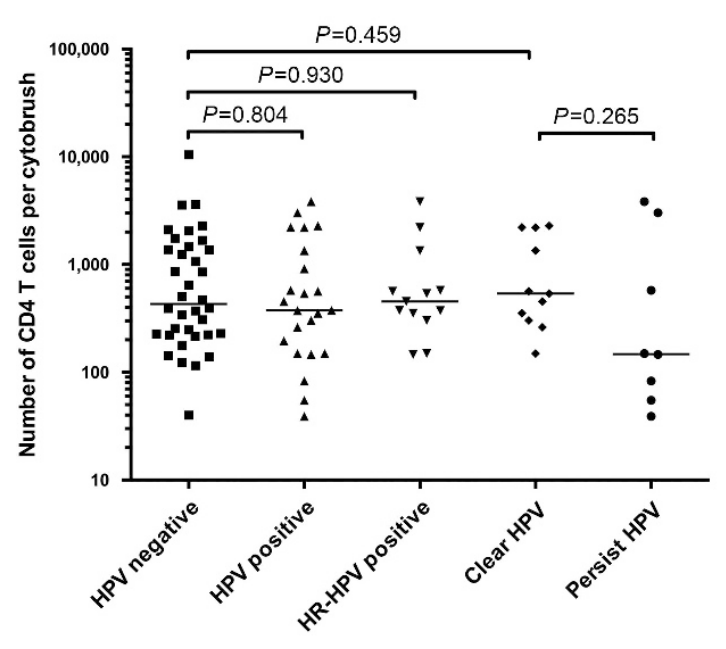

C
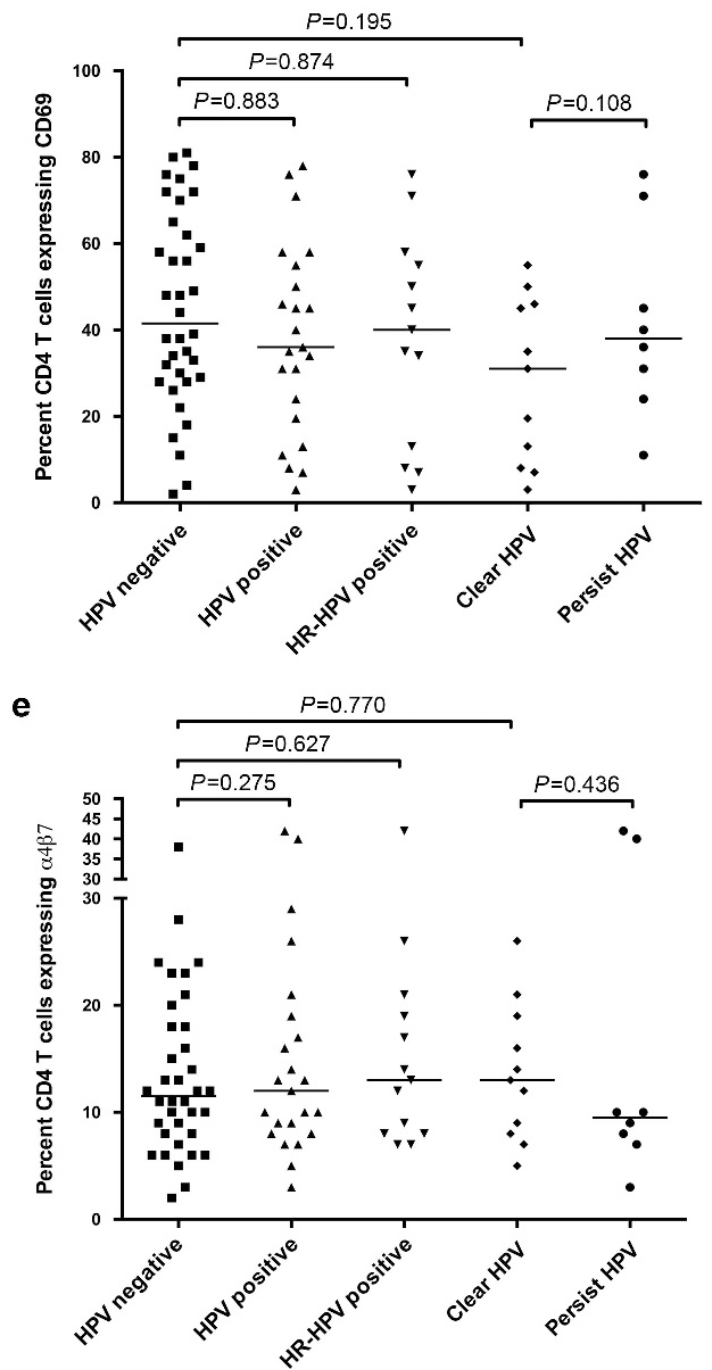

b

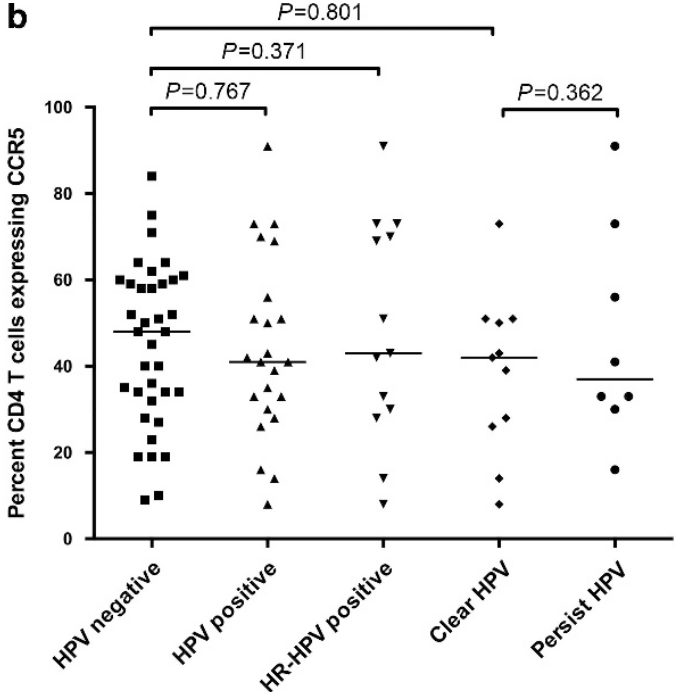

d

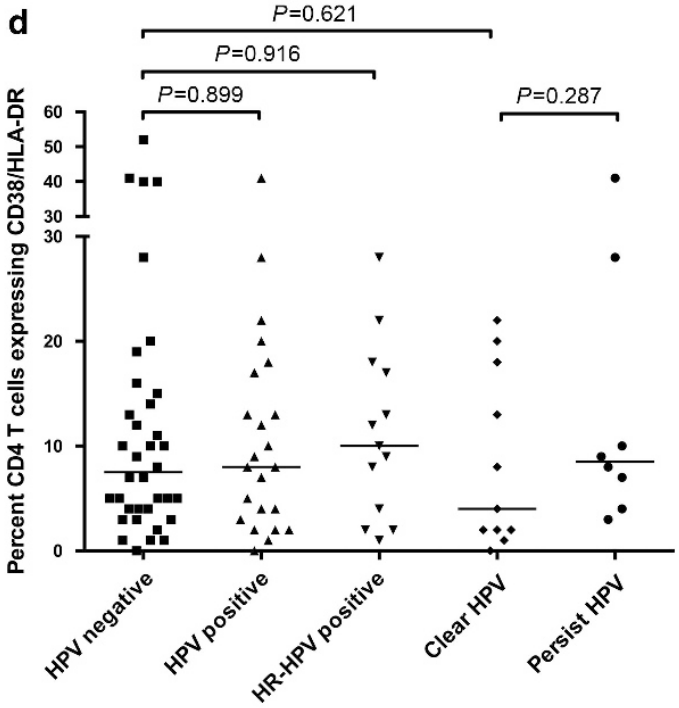

Figure $1 \mathrm{HPV}$ infection status and T cell subsets in the female genital tract. Association of HPV infection as well as HR-HPV infection and subsequent clearance or persistence with the (a) absolute number endocervical CD4 T cells; and the proportion of these cells expressing: (b) CCR5, (c) CD69, (d) CD38 and HLA-DR, and (e) $\alpha 4 \beta 7$. The "HR-HPV Positive", "Clear HPV", and "Persist HPV" groups represent subgroups of the "HPV Positive" group. Statistical comparisons were performed using independent samples $t$-test and confirmed with a multivariate general linear model, with $P$ values for the latter indicated. 

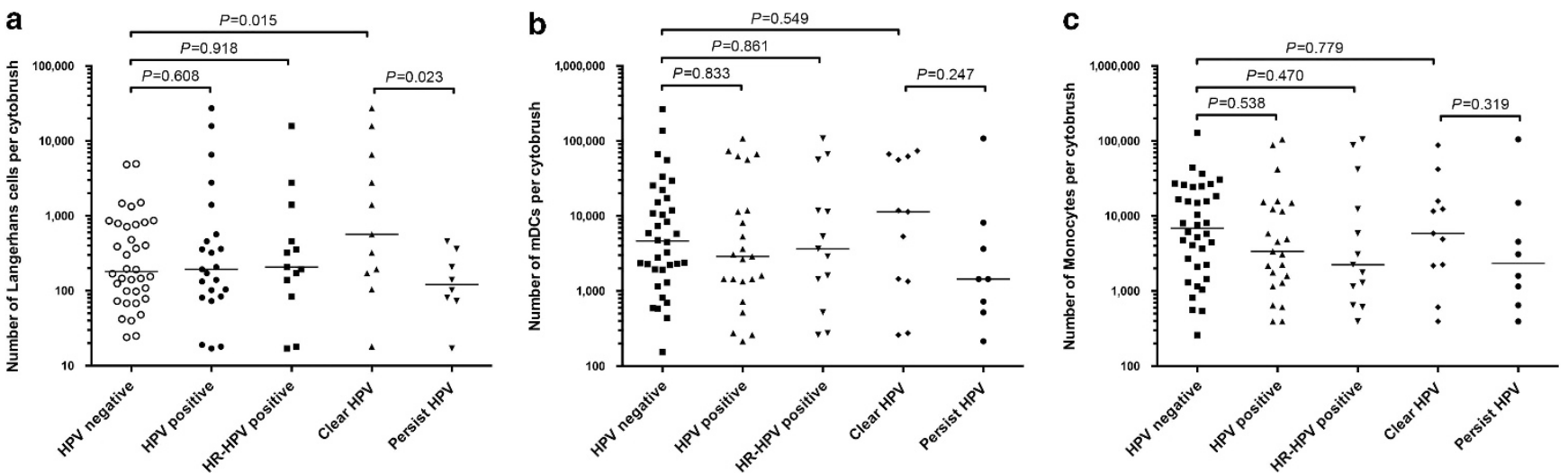

Figure 2 HPV infection status and DC subsets in the female genital tract. Association of HPV and HR-HPV infection as well as subsequent clearance or persistence with the overall number of (a) Langerhans cells, (b) mDCs, and (c) monocytes in the endocervix. The "HR-HPV Positive", "Clear HPV", and "Persist HPV" groups represent subgroups of the "HPV Positive" group. Statistical comparisons were performed using independent samples $t$-test and confirmed with a multivariate general linear model, with $P$ values for the latter indicated.

51 participants were assigned to one of five distinct community state types (CSTs), based on the relative abundance of bacterial taxa as previously described by Gajer et al.: ${ }^{24}$ CST-I (most often dominated by Lactobacillus crispatus), CST-II (most often dominated by Lactobacillus gasseri), CSTIII (most often dominated by Lactobacillus iners), CST-IV (characterized by a paucity of Lactobacillus spp. and a wide array of facultative and strict anaerobes), and CST-V (most often dominated by Lactobacillus jensenii). Overall there was a high correlation between CST distribution and BV status, with $5 / 6(83.3 \%)$ participants with BV (defined as Nugent score $\geqslant 7$ ) classified as CST-IV (Likelihood Ratio $=5.73, P=0.017$ ). The distribution of vaginal CSTs in HPV negative vs. HPV positive women were: $6 / 34$ vs. $1 / 17$ CST-I, $2 / 34$ vs. 0/17 CST-II, 16/34 vs. $6 / 17$ CST-III, $10 / 34$ vs. $10 / 17$ CST-IV; no participants in either group had a microbiome consistent with CST-V (Figure $4 a$ and $\mathbf{b}$ ).

Since CST-IV correlated most closely with a high Nugent score, and since both BV and HPV have been associated with increased HIV acquisition, we examined whether this group had higher rates of HPV infection relative to other CSTs. Interestingly, more participants with HPV infection had a cervico-vaginal microbiome consistent with CST-IV, compared with HPV-negative women (58.8\% vs. $29.4 \%$; $P=0.043$, Figure $4 \mathbf{a}$ and $\mathbf{b}$ ). While significance was lost when postmenopausal women were excluded from the analysis $(P=0.084)$, this likely relates to the reduced sample size, since menopausal status was not associated with CST status or other immune parameters in univariate analysis (data not shown). When we evaluated individual bacterial taxa (Figure 4c and d), participants with HPV had a substantially lower relative abundance of Lactobacillus gasseri $(P=0.009$; Figure 4e), and also of the less abundant bacteria Fusobacterium nucleatum $(P=0.019$; Figure 4f), Cornybacterium accolens $(P=0.001$; Figure 4g), Peptoniphilus harei, Anaerococcus tetradius, Finegoldia magna, and Raoultella planticola (all $P<0.05$; data not shown). Finally,
HPV-infected participants had a higher overall bacterial load (median 9.22log10) than their uninfected counterparts (median 8.95log10, $P=0.018$ ).

\section{DISCUSSION}

HPV infection has been consistently associated with an increased risk of HIV acquisition, ${ }^{4}$ but the underlying biological mechanism(s) behind this association are unclear. We hypothesized that this increased risk might relate to the mucosal recruitment of HIV-susceptible $\mathrm{CD} 4{ }^{+}$target cells, as is seen during chronic HSV-2 infection. ${ }^{18,19,25}$ Furthermore, we hypothesized that this recruitment might be exaggerated in women who go on to clear HPV, since a Th1 cytokine milieu may predominate during host immune clearance ${ }^{5}$ and $\mathrm{Th} 1 \mathrm{CD} 4^{+} \mathrm{T}$ cells are highly HIV susceptible. However, we found neither an association between prevalent HPV infection and genital inflammatory cytokines, nor with endocervical $\mathrm{CD}^{+} \mathrm{T}$ cell numbers or highly susceptible CD4 $+\mathrm{T}$ cell subsets such as those expressing CCR5 or CD69. ${ }^{26}$ Furthermore, among women with prevalent HPV infection, none of these immune parameters differed between participants who subsequently cleared the virus and those who remained persistently infected. However, we did find an increased absolute number of Langerhans cells in women who cleared HPV, and a skewing towards vaginal CST-IV, characterized by a paucity of Lactobacillus spp., in participants with prevalent HPV infection. These results are broadly in keeping with previous reports. ${ }^{27,28}$

Given the consistent association between HPV infection and HIV acquisition, ${ }^{4}$ particularly in the context of host HPV clearance, ${ }^{29}$ we were surprised by the lack of association with both HIV-susceptible target cells and pro-inflammatory cytokines in the female genital tract, although other groups also found no association of HPV or its clearance with elevated pro-inflammatory cytokines. ${ }^{30} \mathrm{HPV}$ infection does not result in cell lysis, and is thought to maintain a regulatory state by evading recognition through the impairment of cytokine signaling. ${ }^{31}$ This state of immune quiescence induced by 

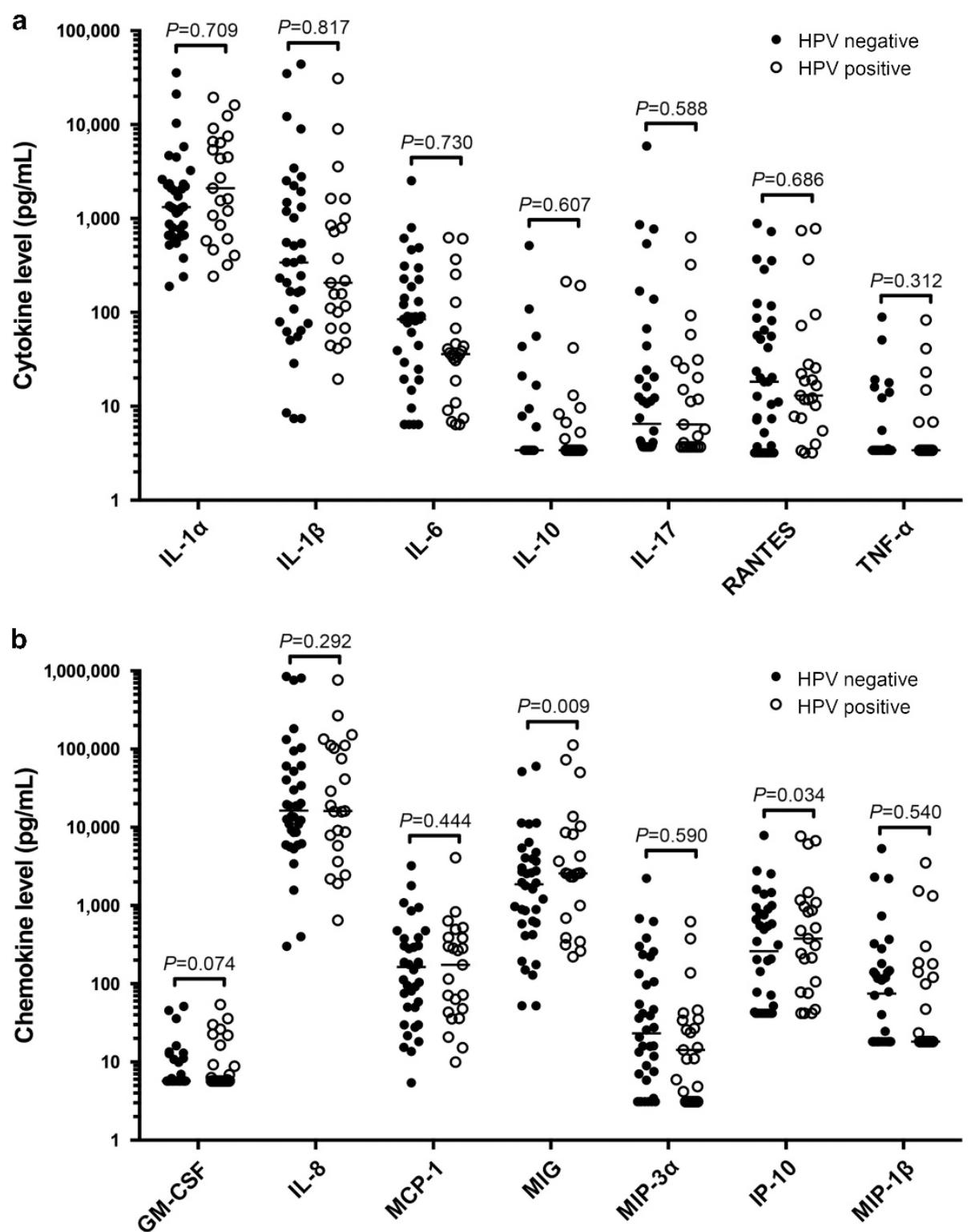

Figure 3 HPV infection and cytokines in the female genital tract. Association between (a) pro-inflammatory cytokine levels and (b) chemokines in undiluted cervicovaginal secretions between HPV negative (full circles) and HPV positive (empty circles) participants. Statistical comparisons were performed using independent samples $t$-test and confirmed with a multivariate general linear model, with $P$ values for the latter indicated.

HPV could explain the lack of both a pro-inflammatory cytokine, as well as $\mathrm{CD} 4 \mathrm{~T}$ cell response to the virus within the local immune milieu of the female genital tract.

Given the lack of association between CD4 T cell subsets and pro-inflammatory cytokines and HPV infection, if the link between HPV infection and HIV acquisition is a causal one, then another mechanism is likely to be at play. It has been suggested that innate immune responses may be vital to clearing HPV infections through recognition via pattern recognition receptors. ${ }^{32}$ Imiquimod, a TLR-7 agonist that induces Langerhans cell recruitment and antigen presentation, increases the clearance of HPV-associated genital warts. ${ }^{33}$ This mirrors our results, with increased numbers of Langerhans cells being associated with HPV clearance. Further, Langerhans cells are reduced in individuals who develop HPV-positive cervical intraepithelial lesions ${ }^{34}$ and a previous study showed that HPV clearance is associated with increased $\mathrm{CDla}^{+}$dendritic cell density in the male foreskin. ${ }^{21}$ Since Langerhans cells may play a direct role in enhancing HIV susceptibility via binding HIV gp120 and facilitating transmission to $\mathrm{CD} 4 \mathrm{~T}$ cells in trans, ${ }^{35}$ this constitutes a potential immune mechanism linking HPV clearance with HIV susceptibility. In addition, HPV-infected participants had elevated genital levels of the chemokines IP-10 and MIG (which were not components of the pro-inflammatory cytokine score). These chemokines are both IFN- $\gamma$ inducible and bind CXCR $3{ }^{36}$ meaning that they may indicate immune activation; interestingly, genital levels of both are reduced in women who remained HIV-uninfected despite frequent HIV exposure, ${ }^{37}$ and 
a
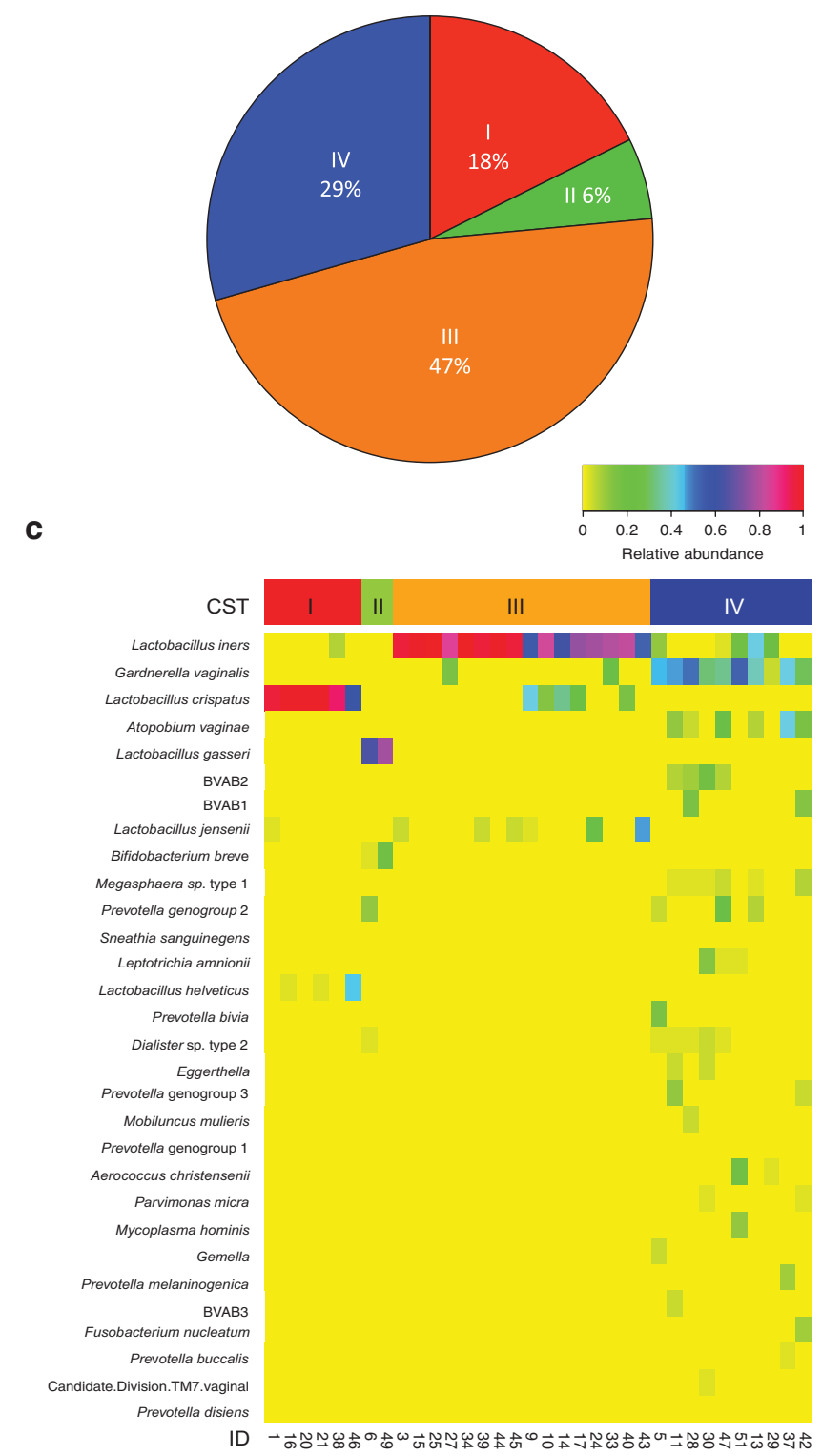

b

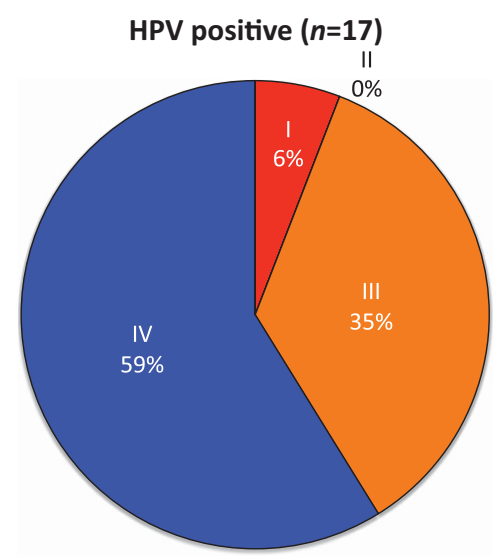

d

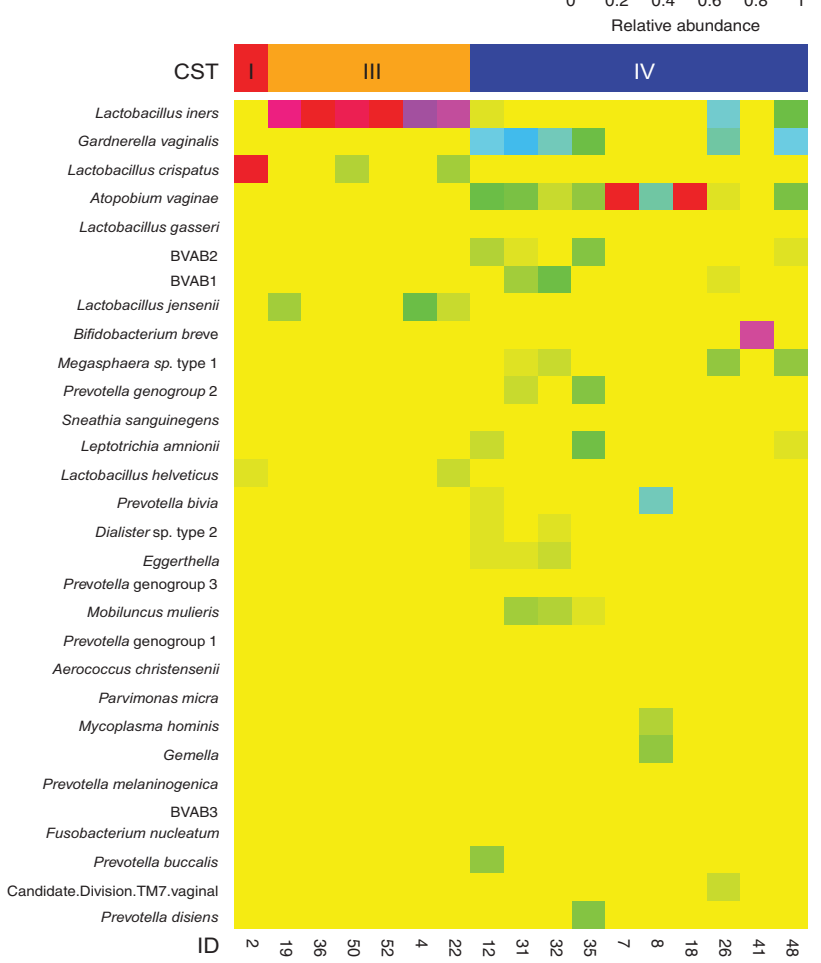

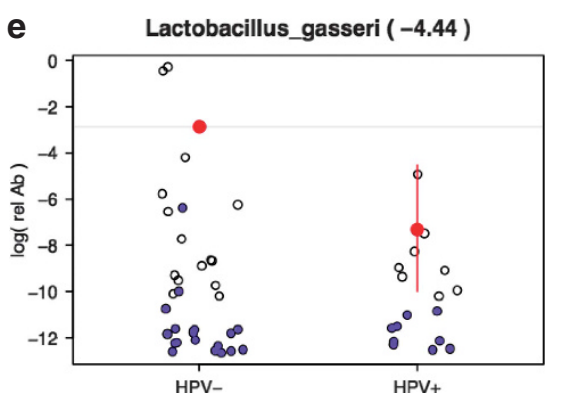
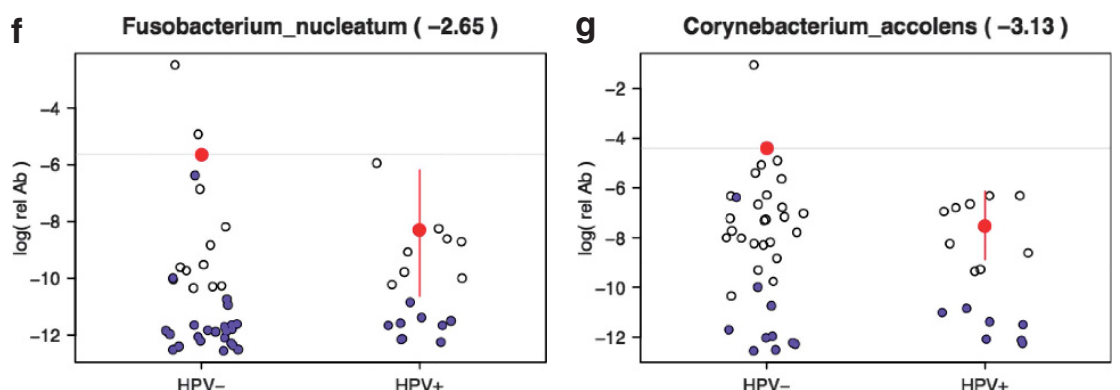

Figure 4 HPV infection and the composition and structure of the vaginal microbiota. The distribution of community state types (CSTs) in (a) HPV negative and (b) HPV positive women, a heatmap of the relative abundance of the 30 most common bacterial phylotypes in (c) HPV negative and (d) HPV positive women grouped by CST distribution, and log relative abundances of specific bacterial phylotypes by HPV status: (e) Lactobacillus gasseri, (f) Fusobacterium nucleatum, (g) Corynebacterium accolens. Samples with relative abundances of 0 were set to a small random number (blue circles). Number in parentheses indicates the difference of log mean relative abundances of each taxa in HPV positive vs. HPV negative participants. Red circles indicate the log of the mean relative abundance of samples with red bars in the HPV positive group indicating the $95 \%$ credible interval of the comparison of the two log mean relative abundances. A significant difference is observed when the $95 \%$ credible interval in the HPV positive group does contain the log mean relative abundance of the HPV negative group (gray line). 
elevated levels of IP-10 were strongly associated with increased HIV acquisition in South African women. ${ }^{38}$ The exact mechanism(s) by which they enhance HIV susceptibility is not known, although we found both to be moderately correlated with an increased numbers of cervical CCR5 ${ }^{+} \mathrm{CD} 4{ }^{+} \mathrm{T}$ cells (both correlation coefficients $0.3, P>0.05$ ).

Furthermore, the vaginal microbiota of participants with HPV infection were more likely to be classified as CST-IV, a state characterized by increased relative abundances of facultative and strict anaerobic bacterial species, ${ }^{39}$ consistent with previous findings demonstrating that women with HRHPV infection have decreased abundance of Lactobacillus spp. ${ }^{27}$ Interestingly, we also observed a decrease in the relative abundance of certain bacterial phylotypes in HPV-infected women, most notably in the generally abundant Lactobacillus gasseri. Other studies have specifically linked L. gasseri with HPV clearance, ${ }^{27}$ and our results support a potential role for these bacteria in defense against HPV. The other bacterial phylotypes that were reduced in the context of HPV infection were of much lower overall abundance and their importance in the genital tract is less clear, although Fusobacterium nucleatum may act as a pathogen. ${ }^{40}$ Although our analysis only found significant reductions in specific bacterial phyla among HPVinfected women, anaerobes as a whole tended to be increased in HPV positive participants, in keeping with a higher proportion of CST-IV. While these differences in the composition and structure of the vaginal microbiota were not associated with alterations in HIV-susceptible CD4 T cells in the female genital tract, they could feasibly play a role in promoting the recruitment of Langerhans cells to sites of HIV exposure, or enhance HIV susceptibility through other mechanisms such as decreased integrity of the vaginal epithelial barrier. ${ }^{41}$

Our study does have limitations, including a relatively modest sample size. While robust associations were demonstrated between HSV-2 serostatus and CD4 ${ }^{+} \mathrm{T}$ cell subsets in a smaller study, ${ }^{19}$ and between HPV infection and specific immune and microbiologic parameters in this one, our sample size could have precluded us from finding true but less robust associations of HPV with other immune parameters. However, any causal effect of HPV infection or its clearance on CD4 ${ }^{+}$ subsets or inflammation would be considerably weaker than the effect of HSV-2. While there was variability in the time of genital sampling in relation to the menstrual cycle and menopause, neither factor was associated with immune parameters or the microbiome in univariate analysis, and their incorporation into our multivariable model did not substantively impact the results. Longitudinal immunological data were not available, with cell populations and cytokines measured only at baseline, and follow up HPV infection data were only obtained after 6 months. Therefore, we may have missed a short-lived immune signature of clearance, and/or immune changes that are induced after (rather than before) HPV clearance. Future studies with more frequent sampling may help to understand the sequence of immune changes that culminates in HPV clearance. Our ability to assess the association of vaginal CST-I/II/V with HPV infection was limited, since these vaginal microbiota types are relatively underrepresented in the ACB community. ${ }^{39}$ Secretions for microbiome analysis were collected by Softcup, and therefore represent a mixture of secretions from the proximal vagina and cervix, while previous studies have used mid-vaginal swabs. However, recent work from our group demonstrates no more variability in the composition of the microbiota as assessed from a cervical and vaginal swab, than was seen between two independent vaginal swabs (Jacques Ravel, personal communication). Finally, limitations in cell numbers obtained by endocervical cytobrush sampling meant that we were not able to assess functional alterations in mucosal $\mathrm{T}$ cells that may be linked to HPV infection or clearance, and it is possible that by focusing on the endocervix our study could miss HPV-associated cellular changes limited to the ectocervix or transformation zone. Future studies that incorporate ectocervical biopsy might be considered to explore this possibility.

In summary, neither cervico-vaginal HPV infection nor $\mathrm{HPV}$ clearance were associated with cervical $\mathrm{T}$ cell alterations or a pro-inflammatory cytokine signature. However, HPV infection was associated with alterations in the composition and structure of the vaginal microbiota and with local elevations in the chemokines IP-10 and MIG, while HPV clearance was associated with increased numbers of cervical Langerhans cell, and each of these could potentially enhance HIV susceptibility. Further research should confirm these observations and characterize the impact of HPV on other immune parameters that may modulate HIV susceptibility.

\section{METHODS}

Participant enrollment and inclusion criteria. Participants were recruited through a flyer at a primary care clinic, Women's Health in Women's Hands Community Health Centre (WHIWH) located in Toronto, Canada. Briefly, ethnic African/Caribbean women living in the Toronto area were recruited, enrolled, and provided informed written consent as per the study protocol approved by the HIV Research Ethics Board at the University of Toronto. Participants both completed a questionnaire and underwent testing for common genital infections. Exclusion criteria for the study included: current infection with HIV-1 or 2/ Neisseria gonorrhoeae, or Chlamydia trachomatis, under 16 years of age, current pregnancy, or reporting a symptomatic genital infection within the previous 3 months.

Study protocol and sampling. Pre-menopausal women visited the clinic 10-18 days after the last day of bleeding from their previous menstrual period. After completing a clinical and sexual behavior questionnaire; blood, urine, and a vaginal swab were collected for STI diagnostics. Instead Softcups (Evofem, San Diego, CA) were used to self-collect undiluted cervico-vaginal secretions for one minute and used for cytokine and microbiota analysis. Two physician-collected endocervical cytobrushes were collected for immune cell phenotyping. Each was gently inserted into the cervical OS, rotated $360^{\circ}$, placed in R10 medium (RPMI 1640 with 10\% heat inactivated fetal bovine serum (Sigma-Aldrich, Carlsbad, CA), $100 \mathrm{mg} \mathrm{mL}^{-1}$ streptomycin, $100 \mathrm{U} \mathrm{mL}^{-1}$ penicillin, and $1 \times$ GlutaMAX-1 (Gibco, Grand Island, $\mathrm{NY}$ ) media), stored at $4{ }^{\circ} \mathrm{C}$, transferred to the lab within 3 hours, filtered $(100 \mu \mathrm{m})$, washed, and divided evenly into two aliquots for staining. Peripheral blood mononuclear cells (PBMCs) isolated by ficoll-hypaque density centrifugation were washed twice in R10 and two 1 million cell aliquots were used for staining. 
Coinfection diagnostics. Gram stain was performed on vaginal smears and evaluated for the presence of yeast and by Nugent criteria for BV diagnostic. ${ }^{42}$ Neisseria gonorrhoeae and Chlamydia trachomatis were detected by nucleic acid amplification test (NAAT; ProbeTech Assay, BD, Sparks, MD) on first-void urine. HerpeSelect gG-1 and gG-2 ELISA (Focus Technologies, Cypress, CA; adjusted threshold of 3.5 [ref. 43] was used to determine participant's HSV-2 serostatus. Selfcollected vaginal swabs were used to identify $37 \mathrm{HPV}$ types using Roche Linear Array (Roche Molecular Systems, Basel, Switzerland) as previously described. ${ }^{44}$ High-risk types were defined as: HPV16,18,31,33,35,39,45,51,52,56,58,59,68 and $69 .^{44}$

Immune cell phenotyping. Endocervical cells collected by cytobrush and PBMCs were stained with two monoclonal antibody panels: one for $\mathrm{T}$ cells and the other DCs. The former consisted of: $\alpha 4$-FITC (Miltenyi Biotec, Bergisch Gladbach, Germany), CD4-ECD (Beckman Coulter, Marseille, France), CCR5-PE, $\beta 7$-APC, CD38-AlexaFluor700, HLA-DR-APC-Cy7, CD69-eFluor450 (BD Biosciences, Franklin Lakes, NJ), Live/Dead Aqua (Invitrogen, Carlsbad, CA), CD25-PerCPCy5.5, CD39-PE-Cy7, and CD3-eFluor650 (eBiosciences, San Diego, CA). The DC panel included: BDCA2-FITC (Miltenyi Biotec), CD207-PE (Beckman Coulter), DC-SIGN-PerCP-Cy5.5, CD206-APC (BD Biosciences), CD83-Streptavidin, CD123-PE-Cy7, CD11cAlexaFluor700, CD14-AlexaFluor780, CD1a-v450, CD3-eFluor650 (eBiosciences), and Live/Dead Aqua (Invitrogen). Cells were enumerated with a BD LSR-2 flow cytometer (BD Systems) and analyzed using FlowJo 9.3.2 software (Treestar, Ashland, OR) by a single, blinded researcher. Immune cell populations from the cervix are reported both as a proportion (\%) and as the total number of cells per cytobrush after the entire contents of cytobrush-collected samples were acquired by flow cytometry (as above). DC subsets were defined as: Myeloid-derived DCs (mDCs; CD3-CD11c ${ }^{+}$), Monocytes (CD3$\mathrm{CD} 14^{+}$), and Langerhans cells (LCs; CD3-CD1a ${ }^{+}$langerin/CD207 ${ }^{+}$). Langerhans cells were gated as per Supplementary Figure 1. Other cellular subsets were gated as previously described. ${ }^{19}$

Cytokine and chemokine assays. Aliquoted and cryopreserved cervico-vaginal secretions were used to assay levels of 14 cytokines (GM-CSF, IL-1 $\alpha$, IL-8, MCP-1, MIG, MIP-3 $\alpha$, RANTES, IL-10, IL-17, IL-1 $\beta$, IL-6, IP-10, MIP-1 $\beta$ and TNF $\alpha$ using the Meso Scale Discovery (MSD; Rockville, MD) electro-chemiluminescent ELISA system. Secretions were plated at $50 \mu \mathrm{l}$ per well and run in duplicate. A standard curve was used to determine the concentration (pg per $\mathrm{ml}$ ). The dilution at which the coefficient of variation exceeded $30 \%$ was defined as the lower limit of quantification. Any sample out of range of the standard curve was repeated following dilution. All samples were assayed and analyzed by blinded lab personnel and the original undiluted concentrations of cytokines in the secretions were calculated.

DNA extraction, 16S rRNA gene amplification and sequencing. Genital secretions $(100 \mu \mathrm{l})$ collected by Instead Softcup (Evofem, San Diego, CA) underwent enzymatic cell wall digest and bead beating for total DNA extraction, as previously described. ${ }^{24,39}$ A dual-barcode system with fusion primers $338 \mathrm{~F}$ and $806 \mathrm{R}$ was used to amplify the V3-V4 regions of the 16S rRNA gene according to Fadrosh et al. ${ }^{45}$ and subsequently sequenced on an Illumina MiSeq instrument (Illumina, San Diego, CA) using the 300 bp paired-end protocol at the Genomic Resource Center at the University of Maryland School of Medicine, Institute for Genome Sciences. If the average phred quality score of 4 consecutive bp was below 15 the sequence reads were trimmed and only retained if their length was at least $75 \%$ of their original length after trimming. FLASH ${ }^{46}$ was used to assemble paired reads. De-multiplexing by binning sequences with the same barcode and quality trimming were performed in QIIME (version 1.8.0) ${ }^{47}$ (see Fadrosh et. al ${ }^{45}$ for additional details). Detection of de novo and reference-based chimera was conducted in UCHIME (v5.1 $)^{48}$ using greengenes database of 16S rRNA gene sequences (Aug, 2013 vers.) ${ }^{49}$ as reference. The processed 16S rRNA gene amplicon sequences were assigned to genera and species, and vaginal microbial communities were assigned to a community state types (CSTs) using taxonomic composition and abundance and the Jensen-Shannon divergence metrics as previously described. ${ }^{24} \mathrm{~A}$ table providing the relative abundance of identified taxa and the CST assignments for each sample was generated and is provided as Supplementary Table S1 with metadata and a legend in Supplementary Tables S2-S3. The 16S rRNA gene sequence data has been deposited into NCBI SRA under accession number SRA362820. Overall bacterial load was measured using an assay previously described by Liu et al. ${ }^{50}$

Statistical analysis. Baseline characteristics and outcomes were summarized with frequencies and proportions for categorical variables and median and range for continuous variables. Differences were assessed using Pearson's $\chi^{2}$ test for categorical variables or an independent samples $t$-test for continuous variables. Cytokine levels and the number of cells per cytobrush were log-transformed to normalize the data (and verified using the Shapiro-Wilk normality test), although the latter is presented in absolute numbers within the text. Univariate immune associations were confirmed in a multivariate general linear model, controlling for demographic factors. Participants producing elevated levels of pro-inflammatory cytokines were defined using a scoring system previously described; ${ }^{11}$ briefly, individuals with elevated levels of pro-inflammatory cytokines were defined as producing at least three out of seven cytokines (IL- $1 \alpha$, IL-8, MIP-3 $\alpha$, RANTES, IL- $1 \beta$, MIP- $1 \beta$, and TNF- $\alpha$ ) in the upper quartile of all participants. Associations between specific bacterial taxa and HPV status were assessed with Bayesian zero-inflated negative binomial models.

All statistical analyses were performed using SPSS version 22 (IBM, New York, NY) and the statistical package R (R Foundation for Statistical Computing, Vienna, Austria).

SUPPLEMENTARY MATERIAL is linked to the online version of the paper at http://www.nature.com/mi

\section{ACKNOWLEDGMENTS}

The investigators acknowledge the kind assistance of the staff at the Women's Help in Women's Hands Clinic, and the time and cooperation of all study participants. This study was supported by the Canadian Institutes of Health Research (RK, grants \#TMI-138656 and \#OCH-131579; BS, studentship) and Ontario HIV Treatment Network (studentship, TJY). RK is supported by a University of Toronto-OHTN Endowed Chair in HIV Research. JR, BM, MSH and PG were supported by the National Institute of Allergy and Infectious Diseases of the National Institute of Health under award number U19AI084044 and R01Al116799. The content is solely the responsibility of the authors and does not necessarily represent the official views of the National Institute of Health.

\section{AUTHOR CONTRIBUTIONS}

All authors have contributed to the study. B.S., W.T., J.R., A.R., and R.K. were involved in the conception and design of the study. B.S., T.J.Y., S.P., P.G., B.M., M.H., J.T., L.C., P.J., M.S., S.H., and K.S. were involved in the execution of the study. B.S., P.G., B.M., M.H., J.R., A.R., and R.K. were involved in the analysis and interpretation of the study.

\section{DISCLOSURE}

The authors declared no conflict of interest.

c 2017 Society for Mucosal Immunology

\section{REFERENCES}

1. Bruni, L., Diaz, M., Castellsague, X., Ferrer, E., Bosch, F.X. \& de Sanjose, S. Cervical human papillomavirus prevalence in 5 continents: meta-analysis of 1 million women with normal cytological findings. J. Infect. Dis. 202, 1789-1799 (2010). 
2. Rosa, M.I., Fachel, J.M., Rosa, D.D., Medeiros, L.R., Igansi, C.N. \& Bozzetti, M.C. Persistence and clearance of human papillomavirus infection: a prospective cohort study. Am. J. Obstet. Gynecol. 199, 617 e611-e617 (2008).

3. Munoz, N. et al. Epidemiologic classification of human papillomavirus types associated with cervical cancer. N. Engl. J. Med. 348, 518-527 (2003).

4. Houlihan, C.F. et al. Human papillomavirus infection and increased risk of HIV acquisition. A systematic review and meta-analysis. AIDS 26, 22112222 (2012).

5. Scott, M., Stites, D.P. \& Moscicki, A.B. Th1 cytokine patterns in cervical human papillomavirus infection. Clin. Diagn. Lab Immunol. 6, 751-755 (1999).

6. Patel, P., Borkowf, C.B., Brooks, J.T., Lasry, A., Lansky, A. \& Mermin, J. Estimating per-act HIV transmission risk: a systematic review. AIDS 28, 1509-1519 (2014).

7. Kaul, R. et al. The genital tract immune milieu: an important determinant of HIV susceptibility and secondary transmission. J. Reprod. Immunol. 77, 32-40 (2008).

8. McKinnon, L.R. et al. Characterization of a human cervical CD4 + T cell subset coexpressing multiple markers of HIV susceptibility. J. Immunol. 187, 6032-6042 (2011).

9. Joag, V.R. et al. Identification of preferential CD4 T-cell targets for HIV infection in the cervix. Mucosal Immunol. 9, 1-12 (2015).

10. Anahtar, M.N. et al. Cervicovaginal bacteria are a major modulator of host inflammatory responses in the female genital tract. Immunity 42, 965-976 (2015).

11. Arnold, K.B. et al. Increased levels of inflammatory cytokines in the female reproductive tract are associated with altered expression of proteases, mucosal barrier proteins, and an influx of HIV-susceptible target cells. Mucosal Immunol. 9, 194-205 (2015).

12. Kaul, R. et al. Genital levels of soluble immune factors with anti-HIV activity may correlate with increased HIV susceptibility. AIDS 22, 2049-2051 (2008).

13. Carnathan, D.G. et al. Activated CD4 + CCR5 + T cells in the rectum predict increased SIV acquisition in SIVGag/Tat-vaccinated rhesus macaques. Proc. Natl. Acad. Sci. USA 112, 518-523 (2015).

14. Masson, L. et al. Defining genital tract cytokine signatures of sexually transmitted infections and bacterial vaginosis in women at high risk of HIV infection: a cross-sectional study. Sex Transm. Infect. 90, 580-587 (2014).

15. Prodger, J.L. et al. Chemokine levels in the penile coronal sulcus correlate with HIV-1 acquisition and are reduced by male circumcision in Rakai, Uganda. Plos Path 12, e1006025 (2016).

16. Levine, W.C. et al. Increase in endocervical CD4 lymphocytes among women with nonulcerative sexually transmitted diseases. J. Infect. Dis. 177, 167-174 (1998).

17. Freeman, E.E., Weiss, H.A., Glynn, J.R., Cross, P.L., Whitworth, J.A. \& Hayes, R.J. Herpes simplex virus 2 infection increases HIV acquisition in men and women: systematic review and meta-analysis of longitudinal studies. AIDS 20, 73-83 (2006).

18. Prodger, J.L., et al. Impact of asymptomatic Herpes simplex virus-2 infection on T cell phenotype and function in the foreskin. AIDS 26, 1319-1322 (2012).

19. Shannon, B. et al. Impact of asymptomatic herpes simplex virus type 2 infection on mucosal homing and immune cell subsets in the blood and female genital tract. J. Immunol. 192, 5074-5082 (2014).

20. Atashili, J., Poole, C., Ndumbe, P.M., Adimora, A.A. \& Smith, J.S. Bacterial vaginosis and HIV acquisition: a meta-analysis of published studies. AIDS 22, 1493-1501 (2008).

21. Tobian, A.A. et al. Human papillomavirus clearance among males is associated with HIV acquisition and increased dendritic cell density in the foreskin. J. Infect. Dis. 207, 1713-1722 (2013).

22. Farhat, S., Nakagawa, M. \& Moscicki, A.B. Cell-mediated immune responses to human papillomavirus $16 \mathrm{E} 6$ and $\mathrm{E} 7$ antigens as measured by interferon gamma enzyme-linked immunospot in women with cleared or persistent human papillomavirus infection. Int. J. Gynecol. Cancer 19, 508-512 (2009).

23. Remis, R.S. et al. The epidemiology of sexually transmitted co-infections in HIV-positive and HIV-negative African-Caribbean women in Toronto. BMC Infect. Dis. 13, 550 (2013).

24. Gajer, P. et al. Temporal dynamics of the human vaginal microbiota. Sci. Transl. Med. 4, 132ra152 (2012).
25. Rebbapragada, A. et al. Negative mucosal synergy between Herpes simplex type 2 and HIV in the female genital tract. AIDS 21, 589-598 (2007).

26. McKinnon, L.R. \& Kaul, R. Quality and quantity: mucosal CD4 + Tcells and HIV susceptibility. Curr. Opin. HIV AIDS 7, 195-202 (2012).

27. Brotman, R.M. et al. Interplay between the temporal dynamics of the vaginal microbiota and human papillomavirus detection. J. Infect. Dis. 210, 1723-1733 (2014).

28. Dareng, E.O. et al. Prevalent high-risk HPV infection and vaginal microbiota in Nigerian women. Epidemiol. Infect. 144, 123-137 (2016).

29. Smith-McCune, K.K. et al. Type-specific cervico-vaginal human papillomavirus infection increases risk of HIV acquisition independent of other sexually transmitted infections. PLOS ONE 5, e10094 (2010).

30. Kriek, J.M. et al. Female genital tract inflammation, HIV co-infection and persistent mucosal Human Papillomavirus (HPV) infections. Virology 493, 247-254 (2016).

31. Kaul, R. \& Hirbod, T. Genital epithelial cells: foot soldiers or fashion leaders?. J. Leukoc. Biol. 88, 427-429 (2010).

32. Daud, II, Scott, M.E., Ma, Y., Shiboski, S., Farhat, S. \& Moscicki, A.B. Association between toll-like receptor expression and human papillomavirus type 16 persistence. Int. J. Cancer 128, 879-886 (2011).

33. Beutner, K.R., Spruance, S.L., Hougham, A.J., Fox, T.L., Owens, M.L. \& Douglas, J.M. Jr. Treatment of genital warts with an immune-response modifier (imiquimod). J. Am. Acad. Dermatol. 38 (2 Pt 1), 230-239 (1998).

34. Mota, F., Rayment, N., Chong, S. \& Singer, A. Chain B. The antigenpresenting environment in normal and human papillomavirus (HPV)related premalignant cervical epithelium. Clin. Exp. Immunol. 116, 33-40 (1999).

35. Turville, S.G. et al. HIV gp120 receptors on human dendritic cells. Blood 98, 2482-2488 (2001).

36. Hsieh, M.F. et al. Both CXCR3 and CXCL10/IFN-inducible protein 10 are required for resistance to primary infection by dengue virus. J. Immunol. 177, 1855-1863 (2006).

37. Lajoie, J. et al. A distinct cytokine and chemokine profile at the genital mucosa is associated with HIV-1 protection among HIV-exposed seronegative commercial sex workers. Mucosal Immunol. 5, 277-287 (2012).

38. Masson, L. et al. Genital inflammation and the risk of HIV acquisition in women. Clin. Infect. Dis. 61, 260-269 (2015).

39. Ravel, J. et al. Vaginal microbiome of reproductive-age women. Proc. Natl. Acad. Sci. USA 108 (Suppl 1), S4680-S4687 (2011).

40. Citron, D.M. Update on the taxonomy and clinical aspects of the genus fusobacterium. Clin. Infect. Dis. 35 (Suppl 1), S22-S27 (2002).

41. Borgdorff, H. et al. Cervicovaginal microbiome dysbiosis is associated with proteome changes related to alterations of the cervicovaginal mucosal barrier. Mucosal Immunol. 9, 621-633 (2016).

42. Nugent, R.P., Krohn, M.A. \& Hillier, S.L. Reliability of diagnosing bacterial vaginosis is improved by a standardized method of gram stain interpretation. J. Clin. Microbiol. 29, 297-301 (1991).

43. Biraro, S., Mayaud, P., Morrow, R.A., Grosskurth, H. \& Weiss, H.A. Performance of commercial herpes simplex virus type-2 antibody tests using serum samples from Sub-Saharan Africa: a systematic review and meta-analysis. Sex Transm. Dis. 38, 140-147 (2011).

44. Shannon, B. et al. Community-specific Prevalence and Type Distribution of HPV Infection among African/Caribbean Women in Toronto, Canada (Abstract PH.PP02.82). 29th International Papillomavirus Conference (Seattle, WA, 2014)..

45. Fadrosh, D.W. et al. An improved dual-indexing approach for multiplexed 16 SrRNA gene sequencing on the lllumina MiSeq platform. Microbiome 2, 6 (2014).

46. Magoc, T. \& Salzberg, S.L. FLASH: fast length adjustment of short reads to improve genome assemblies. Bioinformatics 27, 2957-2963 (2011).

47. Caporaso, J.G. et al. QIIME allows analysis of high-throughput community sequencing data. Nat. Methods 7, 335-336 (2010).

48. Edgar, R.C., Haas, B.J., Clemente, J.C., Quince, C. \& Knight, R. UCHIME improves sensitivity and speed of chimera detection. Bioinformatics 27 , 2194-2200 (2011).

49. McDonald, D. et al. An improved Greengenes taxonomy with explicit ranks for ecological and evolutionary analyses of bacteria and archaea. ISMEJ. 6, 610-618 (2012).

50. Liu, C.M. et al. BactQuant: an enhanced broad-coverage bacterial quantitative real-time PCR assay. BMC Microbiol. 12, 56 (2012). 